\title{
Spatial and temporal variation in the generation time of a bird metapopulation: density regulation and the evolutionary potential of a pace-of-life measure
}

\author{
Yimen Araya-Ajoy ${ }^{1}$, Alina Niskanen ${ }^{2}$, Peter Ranke ${ }^{1}$, Hannah Froy ${ }^{1}$, Thomas Kvalnes ${ }^{1}$, \\ Bernt Rønning ${ }^{1}$, Michael Pedersen ${ }^{1}$, Thor-Harald Ringsby ${ }^{1}$, Henrik Jensen ${ }^{1}$, Bernt-Erik \\ Sæther $^{3}$, and Jonathan Wright ${ }^{4}$ \\ ${ }^{1}$ Norwegian University of Science and Technology \\ ${ }^{2}$ University of Oulu \\ ${ }^{3}$ Centre for Biodiversity Dynamics \\ ${ }^{4} \mathrm{NTNU}$
}

November 20, 2020

\begin{abstract}
Generation time determines the pace of key demographic and evolutionary processes. Quantified as the weighted mean age at reproduction, it can be studied as a trait that varies within and among populations and may evolve in response to ecological conditions. We combined quantitative genetic analyses with age- and density-dependent models to study generation time variation in a bird metapopulation. Generation time was heritable, and males had longer generation times compared with females. Individuals with longer generation times had a higher lifetime reproductive success but not a higher expected population growth rate. Density regulation acted on recruit production, suggesting that longer generation times should be favored when populations are closer to carrying capacity. Furthermore, generation times were shorter when populations were growing, and longer when populations were closer to equilibrium or declining. These results support classic theory predicting that density regulation is an important driver of the pace of life-history strategies.
\end{abstract}

\section{Hosted file}

Main text v2.pdf available at https://authorea.com/users/334319/articles/494181-spatial-andtemporal-variation-in-the-generation-time-of-a-bird-metapopulation-density-regulationand-the-evolutionary-potential-of-a-pace-of-life-measure 